MATEC Web of Conferences 3, 01011 (2013)

DOI: $10.1051 /$ matecconf $/ 20130301011$

(c) Owned by the authors, published by EDP Sciences, 2013

\title{
Second harmonic generation: applications in phase diagram investigations
}

\author{
F. Simon, J. Mahieux, S. Clevers, N. Couvrat, V. Dupray, and G. Coquerel
}

PRES Normandie, Crystallogenesis Unit, SMS, EA 3233 IMR 4114 Université de Rouen, F-76821 Mont-Saint-Aignan Cedex, France

\section{Introduction}

Second harmonic generation (SHG) is a nonlinear optical effect occurring in crystals with non-centrosymmetric space groups [1]. The second harmonic generation efficiency of a given material depends mainly on (i) the intrinsic crystallographic features of the phase, (ii) the orientation of the crystal(s) (crystallographic axes) with reference to the fundamental beam orientation and polarization, (iii) the crystal size distribution. SHG was formerly proposed as a rapid, reliable and very sensitive test of non-centrosymmetry for powdered crystalline compounds [2] with applications to structure assignments and pre-screening of conglomerates [3]. It was also proposed as an efficient technique for the quantification of a crystalline phase inside an amorphous matrix [4] and, recently, we demonstrated that SHG can be used to assess the structural purity of an organic compound down to the ppm level [5].

Herein we investigate the SHG efficiency of several binary organic powder mixtures (recrystallized from a solvent) exhibiting eutectic phase behavior.

\section{Experimental}

The binary system of atropoisomers (-) Bis-Tetralone / (+) Bis-Tetralone was considered. This system crystallizes as a conglomerate forming system [6].

Powder samples at various compositions were dissolved and crystallized from acetone at room temperature in $9 \mathrm{~mL}$ glass vials.

Thermal analyses of the powder samples (mass of ca. $10 \mathrm{mg}$ ) were conducted on DSC 204 F1 (Netzsch). The data were processed by using the Netzsch-TA Proteus ${ }^{\circledR}$ Software v 4.8.4.).

The phases were analyzed by XRPD (D8 Discover, Bruker) and diffractograms were compared to reference diffractograms (CSD database, CCDC) in order to assess the crystallographic nature of the polymorphs .

For the system studied, the SHG activity of the powder samples obtained for different compositions was measured using the Kurtz and Perry method with the experimental setup previously described by Galland et al.[3]

Binary phase diagram were constructed by using DSC and XRPD.

The enthalpy of the eutectic invariant was calculated (integration of the corresponding peak) for each sample in order to draw the Tammann graph.

\section{Results}

The results (Figure 1) show that a maximal SHG efficiency is obtained for the powder mixture at the eutectic composition. The evolution of the SHG efficiency versus the composition is closely similar to the Tammann graph.

This can be explained by the presence of microstructures which strongly enhance the signal. Indeed, eutectic solidification often leads to particular microstructures with both chemical entities (e.g lamellar, rod-like, etc.) [7]. SHG signal arising from this type of particles is often enhanced due to the possibility of quasiphase-matching (optical effect in which the destructive interference between both fundamental wave and second harmonic wave is avoided) [8] 

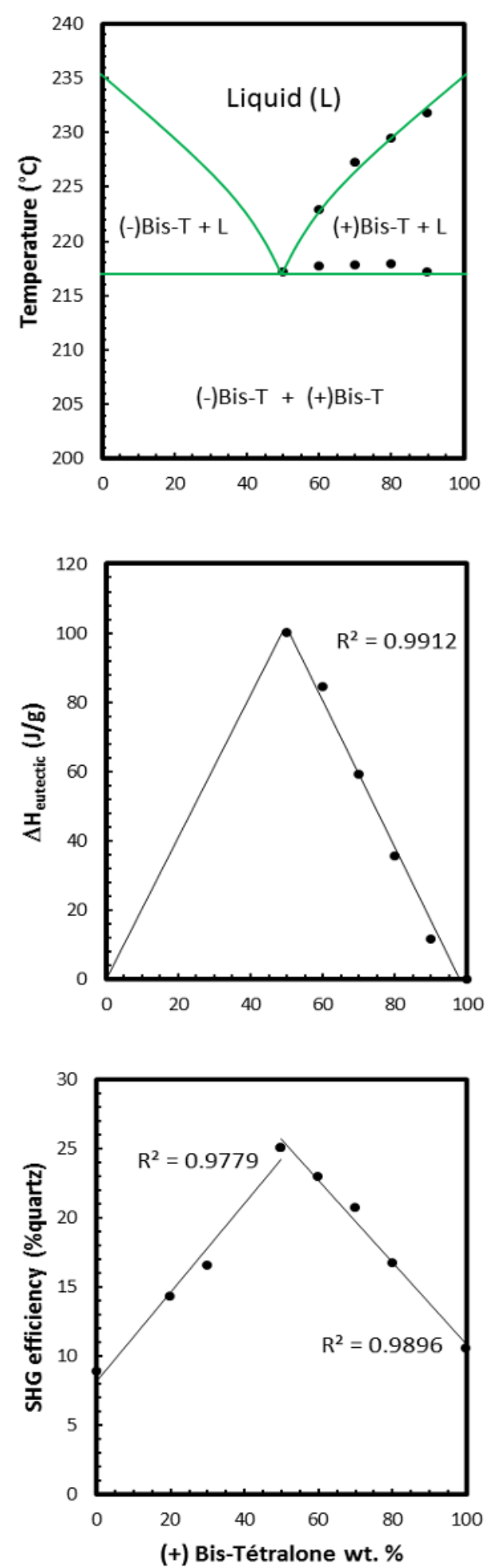

Figure 1. (a) Temperature v.s. composition phase equilibrium. Liquidus lines were constructed with the maximal deflection of the fusion peak and eutectic invariants were constructed with onset eutectic endotherms. Solid lines correspond to stable equilibria, dashed lines correspond to metastable equilibria and dotted lines correspond to suspected solvus (when presence of solid solution). (b) Enthalpy v.s. composition plot (Tammann graph). (c) SHG efficiency v.s. composition plot. SHG intensities are normalized by tacking as a reference unit of the

SHG efficiency of quartz (44 microns). Uncertainties on measurements (volume, sample weight, fluctuation of the laser) have not been drawn for clarity but are evaluated at less than

\section{Conclusion}

The results show a high similarity between data given by thermal analysis (Tammann graph) and nonlinear optics (SHG).

This study highlights the potential of SHG in phase diagram investigation. Moreover, SHG can also of help in the screening of new composite materials for frequency conversion applications.

\section{References}

1. N. Bloembergen, Nonlinear Optics, World Scientific Publishing Co. Pte. Ltd. (1996)

2. S.K. Kurtz and T.T. Perry, J. Appl. Phys. 393798 (1968)

3. A. Galland, V. Dupray, B. Berton, S. Morin-Grognet, M. Sanselme, H. Atmani, G. Coquerel, Cryst. Growth Des. 92713 (2009)

4. D. Wanapun, U.S. Kestur, D.J. Kissick, G.J. Simpson, L.S. Taylor, Anal. Chem. 825425 (2010)

5. S. Clevers, F. Simon, V. Dupray, G. Coquerel, J. Therm. Anal. Calorim. (2012) DOI 10.1007/s10973-012-2763-y

6. J. Mahieux, M. Sanselme, S. Harthong, C. Melan, C. Melan, C. Aronica, L. Guy, G. Coquerel, (to be published)

7. W.D. Callister, Fundamentals of Materials Science and Enginnering, John Wiley \& Sons, Inc. (2001)

8. J.A. Armstrong, N. Bloembergen, J. Ducuing, P.S. Pershan, Phys. Rev. 1271918 (1962) $15 \%$ 